\title{
Moxonidina. Um Novo Anti-Hipertensivo de Ação Central
}

\author{
Carolina Garcia Soares Leães, Guido Aranha Rosito \\ PortoAlegre, RS
}

\begin{abstract}
A hipertensão arterial sistêmica (HAS) é provavelmente o maior problema da saúde pública mundial, por sua contribuição significativa na morbimortalidade de doenças cardiovasculares ${ }^{1,2}$. Sua frequiência aumenta, paralelamente, com a idade da população, atingindo 45 a $50 \%$ na faixa etária de 65 anos ${ }^{1}$. O tratamento da HAS visa a redução da pressão arterial (PA) e a diminuição da morbimortalidade ${ }^{1}$, através da reeducação do paciente e do uso de fármacos anti-hipertensivos ${ }^{3}$.

Neste trabalho fez-se uma revisão de aspectos clínicos e farmacológicos a respeito do novo anti-hipertensivo de ação central, a moxonidina. Ela atua como agonista parcial altamente seletivo para os receptores $\mathrm{I}_{1}$ ligando-se de forma competitiva e, com enorme afinidade, na porção rostralventrolateral do bulbo $(\mathrm{VLM})^{4}$, principal área responsável pela manutenção da PA e de reflexos vasomotores ${ }^{5,6}$, possuindo receptores $\mathrm{a}_{2}$ adrenérgicos e $\mathrm{I}_{1}$ imidazolínicos ${ }^{7}$.
\end{abstract}

\section{Aspectos farmacodinâmicos}

A moxonidina é um agonista parcial e seletivo dos receptores $\mathrm{I}_{1}$ cuja ligação é competitiva e de alta afinidade ${ }^{5}$. A ativação desses receptores no VLM demonstrou reduzir a atividade do sistema nervoso simpático e, assim, diminuir a $\mathrm{PA}^{8,9}$, sugerindo que o efeito hipotensor da moxonidina decorra, preferencialmente, da ação nesses receptores $I_{1}$ bulbares, causando uma diminuição do tônus simpático ${ }^{6} \mathrm{e}$ da resistência vascular periférica (RVP) (fig. 1) ${ }^{5,10-13}$. Esta afinidade é muito maior com os receptores imidazolínicos do que com os $\mathrm{a}_{2}$ adrenérgicos ${ }^{14}$, sendo descritas diferenças entre $40{ }^{5}$ e 600 vezes ${ }^{14}$. A seletividade varia com os ligantes e com as preparações teciduais utilizados ${ }^{14}$.

Sugeriu-se esta maior seletividade pelos receptores $\mathrm{I}_{1}$ através da verificação de uma maior supressão do efeito hipotensivo da moxonidina pelo idaxozan (antagonista $\mathrm{I}_{1}$ ) do que pela ioimbina (antagonista $\left.\alpha_{2}\right)^{15}$. A não correlação entre receptores $\mathrm{a}_{2}$ e diminuição da PA confronta a teoria aceita, até recentemente, sobre o mecanismo de ação dos anti-hipertensivos centrais ${ }^{5}$. Estudos demonstraram que apenas parte da ação anti-hipertensiva central da clonidina

Fundação Faculdade Federal de Ciências Médicas de Porto Alegre Correspondência: Guido A. Rosito - Fundação Faculdade Federal de Ciências Médicas de PA - Rua Sarmento Leite, 245 - $3^{\circ}$ - 90050-170 - Porto Alegre, RS Recebido para publicação em 5/1/98

Aceito em 24/3/98 era bloqueada pela ioimbina (antagonista $\alpha_{2}$ ), e parte pelo idaxozan (antagonista $\left.I_{1}\right)^{15}$. Além disso, foram testados compostos imidazólicos em animais, verificando-se também uma correlação entre afinidade pelos receptores $I_{1}$ no VLM e dose necessária para o controle da $\mathrm{PA}^{5}$. Este efeito hipotensor demonstrou, ainda, ser totalmente revertido pela administração de efaroxan (antagonista seletivo $\mathrm{I}_{1}$ ) após a injeção bilateral de moxonidina no $\mathrm{VLM}^{7}$. Todavia, há relatos de que a administração sistêmica de um antagonista $a_{2}$ seletivo bloqueia por completo o efeito hipotensor de uma dose subseqüente de moxonidina ${ }^{6}$.

\section{Aspectos farmacocinéticos}

A moxonidina é rapidamente absorvida $\mathrm{VO}$ e possui uma biodisponibilidade de quase $90 \%$ em estudos feitos em voluntários sadios e hipertensos com função renal normal $^{7,14,16}$. Sua absorção não perece ser alterada pela ingestão de alimentos ${ }^{14}$. Atinge um pico plasmático de concentração em $1 \mathrm{~h}$, sendo na maior parte (58-60\%) excretada sob forma inalterada por via renal ${ }^{10,15}$, com biotransformação hepática não significativa ${ }^{7}$. Possui uma meia-vida $(\mathrm{t} 1 / 2)$ de 2,6h em pacientes com função renal normal, que aumenta em pacientes com insuficiência renal para $3,5 \mathrm{~h}$ (taxa de filtração glomerular(TFG) $30 \mathrm{a} 60 \mathrm{~mL} / \mathrm{min}$ ) ou $6,9 \mathrm{~h}$ (TFG < $30 \mathrm{~mL} / \mathrm{min})^{17}$. Uma pequena fração da droga $(7 \%)$ liga-se a proteínas plasmáticas ${ }^{14,17}$. Não há evidências de acúmulo de moxonidina no organismo em tratamento crônico ${ }^{7}$, ou em uma semana de tratamento com $0,3 \mathrm{mg} / \mathrm{dia}$ em pacientes com insuficiência renal severa ${ }^{17}$. O efeito hipotensor máximo da moxonidina se dá $2 \mathrm{a} 4 \mathrm{~h}$ após seu pico plasmático ${ }^{7}, \mathrm{e}$ se prolonga até $12 \mathrm{~h}$ após a sua administração ${ }^{14}$. Esta diferença entre o tempos de meia vida e de ação desta droga sugere uma retenção da moxonidina no SNC ${ }^{11}$, com liberação mais lenta da droga do sítio de ação, ou reflete a redução retardada nos níveis plasmáticos de adrenalina ${ }^{14}$, que ocorre após 4 a $6 \mathrm{~h}$ da administração deste fármaco ${ }^{10,16,17}$. A moxonidina encontra-se no leite materno em uma concentração 50 a $100 \%$ maior que no plasma ${ }^{14}$. Seus padrões farmacocinéticos seguem a característica geral de um modelo de único compartimento ${ }^{18}$ (tab. I).

\section{Efeitos desejáveis}

Efeito da moxonidina na HAS - Dois estudos não comparativos feitos em pacientes com hipertensão essencial leve a moderada, tratados por um ano com moxonidina, de- 


MOXONIDINA
$\Downarrow \oplus$
RECEPTORES IMIDAZOLÍNICOS I NO VLM
$\downarrow_{\theta}$
SISTEMA NERVOSO SIMPÁTICO
$\Downarrow$
$\downarrow$ DA LIBERAÇÃO DECATECOLAMINAS
$\Downarrow$
$\downarrow$ DA RVP
$\Downarrow$
$\downarrow$ DA PRESSÃO ARTERIAL
$\oplus=$ Estimulação $/ \theta=$ Inibição $/ \downarrow=$ Diminuição $/$
RVP $=$ Resistência Vascular Periférica

Figura 1. Mecanismo de ação da Moxonidina.

monstraram alta eficácia anti-hipertensiva: no $1^{\circ}$ mais de $80 \%$ dos pacientes tratados com doses entre 0,2 e $0,6 \mathrm{mg} / \mathrm{dia}$ obtiveram normalização da PA (diastólica (PAD) $<90 \mathrm{mmHg}$ ), ou redução >10 $\mathrm{mmHg}$ na PAD em repouso (fig. 2 e 3$)^{8}$ e, no $2^{\circ}$, em 60 a $70 \%$ dos pacientes tratados com uma média de $0,3 \mathrm{mg} / \mathrm{dia}$ houve redução da PAD a valores $<90 \mathrm{mmHg}{ }^{14}$. Observou-se, ainda, uma melhor resposta em idosos ${ }^{8}$. Além disto, estudos controlados por placebo após dose única ou tratamento crônico com moxonidina $(0,2 \mathrm{a}$ $0,4 \mathrm{mg} \mathrm{VO}$ ) demonstraram uma redução significativa e tempo dependente nas pressões sistólica (PAS) e PAD ${ }^{17,19}$, tanto em repouso como durante o exercício ${ }^{10,14}$. Todavia, em estudos com animais, demonstrou-se que efeito anti-hipertensivo da moxonidina administrada via IV é precedido por uma elevação transitória da PA, provavelmente resultante da estimulação dos receptores $\mathrm{a}_{2}$ periféricos ${ }^{14}$.

Em um ensaio clínico controlado por placebo, em que se realizou monitorização ambulatorial da pressão arterial (MAPA), verificou-se eficácia da moxonidina na redução da PA nos regimes $0,2 \mathrm{mg} 2 \mathrm{x} / \mathrm{dia}$ e $0,4 \mathrm{mg} 1 \mathrm{x} / \mathrm{dia}$, sendo que o último apresentou uma variação dia/noite mais fisiológica da $\mathrm{PA}^{17}$. Outro estudo aberto, utilizando a MAPA em 22 pacientes hipertensos, também demonstrou uma redução na PA após 12 semanas de tratamento com 0,2 a $0,6 \mathrm{mg}$ de moxonidina (fig. 4). Verificou-se, ainda, que o pico hipertensivo matinal foi bloqueado mesmo após $12 \mathrm{~h}$ da administração da última dose ${ }^{17}$.

Efeitos hemodinâmicos - Estudos não comparativos com moxonidina em dose única $(0,2$ a 0,25 ou $0,4 \mathrm{mg})$, ou após quatro semanas de tratamento $(0,37 \mathrm{mg} / \mathrm{dia})$, não demonstraram alteração relevante no débito cardíaco (DC) em humanos ${ }^{10,14}$. Não há evidência de efeito significativo na freqüência cardíaca $(\mathrm{FC})$ em tratamento prolongado com doses hipotensoras desta droga ${ }^{8}$. A pressão da artéria pulmonar não se alterou após dose única ${ }^{10,14} \mathrm{ou}$ administração repetida de moxonidina ${ }^{14}$.

Efeitos neuro-humorais - Estudos em humanos têm demonstrado diminuição dos níveis de renina e noradrenalina em repouso (23-35\% e 25-35\%, respectivamente) e exercício (19\% e 25\%) após $4 \mathrm{a}$ 6h da administração única de moxonidina $0,4 \mathrm{mg}^{7,14}$. Os níveis plasmáticos de noradrenalina não se alteraram nas primeiras $4 \mathrm{~h}$ decorrentes da administração da droga, mas houve redução de $27 \%$ em 6 h e de $39 \% \mathrm{em} 9 \mathrm{~h}^{14}$. Um estudo controlado por placebo também relatou diminuição nos níveis plasmáticos de noradrenalina, adrenalina e renina após 4 a $6 \mathrm{~h}$ da administração de moxonidina $(0,2 \mathrm{a} 0,4 \mathrm{mg})^{17}$. Após quatro semanas de tratamento

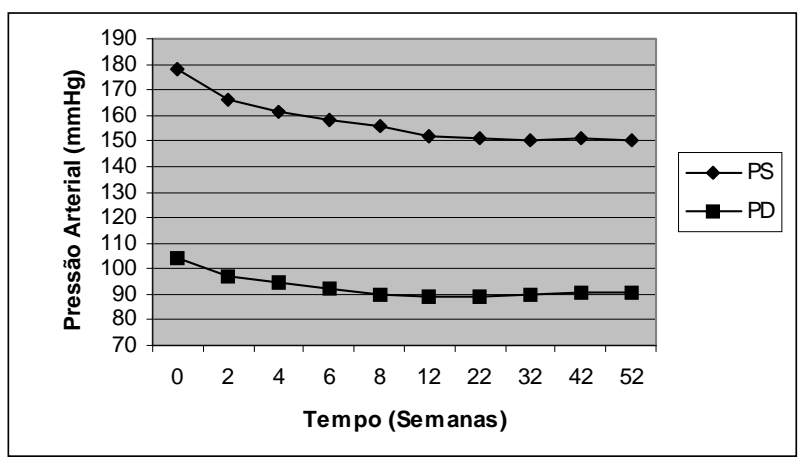

Fig. 2 - Efeito da moxonidina nas pressões sistólica (PS) e diastólica (PD) durante 52 semanas de tratamento. Adaptado da ref $^{7}$.

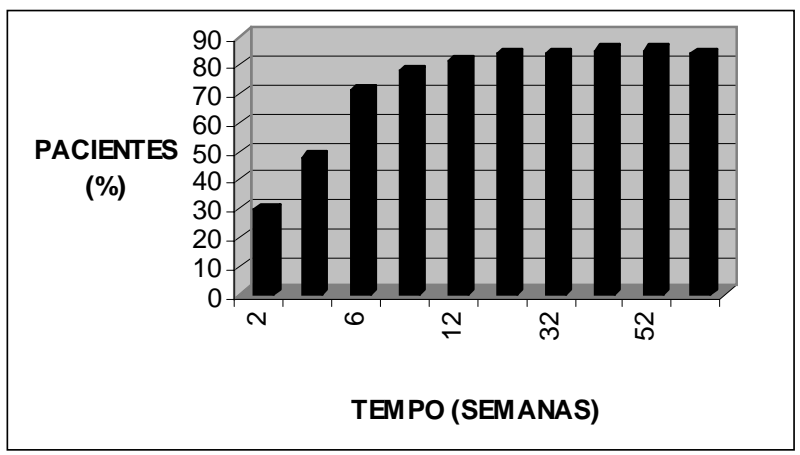

Fig. 3 - Grau de resposta ao tratamento com moxonidina por 52 semanas (resposta = $\mathrm{PD}<90 \mathrm{mmHg}$ ou redução de no mínimo $10 \mathrm{mmHg}$ na PD). Adaptado da ref ${ }^{8}$.

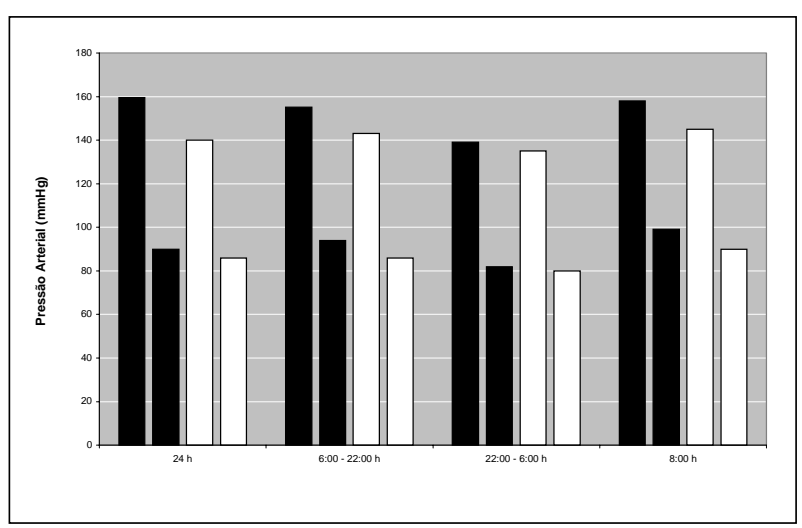

Fig. 4 - Médias das pressões sistólicas (S) e diastólica (D) obtidas com a MAPA antes (colunas escuras) e após (coluna brancas) 12 semanas de terapia com 0,2mg a 0,6mg/ dia de moxonidina. Adaptado da ref ${ }^{17}$. 
(0,4mg de moxonidina), manteve-se a redução de renina, mas não se observou mais diferença significativa nos níveis de catecolaminas ${ }^{17}$.

Observaram-se, ainda, redução significativa do fator atrial natriurético (FAN) durante o exercício (23\%) ${ }^{11,14}$ e elevações transitórias nos níveis de GH após dose única de moxonidina ( 0,4 e $0,3 \mathrm{mg}$, respectivamente). Não foram verificadas alterações relevantes nos níveis de prolactina, TSH, gonadotrofinas, hormônios adrenocorticotróficos ${ }^{7,11}$ e dopamina ${ }^{14}$.

Efeito na estrutura cardiovascular - Há evidências de que a moxonidina causa regressão da hipertrofia do ventrículo esquerdo (VE) em humanos e em animais hipertensos ${ }^{15}$. Com efeito, esta droga mostrou diminuir em aproximadamente $30 \%$ a hipertrofia do VE em pacientes hipertensos submetidos a tratamento prolongado ${ }^{17}$. Estudos comparativos entre nifedipina e moxonidina em animais e em humanos demonstraram que ambas diminuem o grau de hipertrofia do VE durante tratamento de três a seis meses ${ }^{14,20}$. Em humanos, a redução foi de $15 \%{ }^{20}$.

Outro experimento feito em animais demonstrou que todas as alterações da estrutura cardíaca observadas em animais hipertensos não tratados (hipertrofia VE, necrose de miócitos fibrose miocárdica, fibrose perivascular) foram revertidas parcial ou totalmente com administração de moxonidina durante três a seis meses $(8 \mathrm{mg} / \mathrm{kg} / \mathrm{dia})^{4}$. Em estudo realizado em pacientes com angina microvascular hipertensiva, em tratamento prolongado com moxonidina (oito a 12 meses com uma dose de 0,4 a $0,8 \mathrm{mg} /$ dia), houve diminuição da espessura da camada média das arteríolas intramurais (favorecendo o fluxo coronário), aumento da densidade capilar, diminuição da fibrose perivascular, regressão da hipertrofia de VE e aumento da função endotelial ${ }^{21}$.

\begin{tabular}{|lc|}
\hline \multicolumn{2}{|c|}{ Tabela I - Aspectos farmacocinéticos da moxonidina } \\
\hline Farmacocinética & Moxonidina \\
\hline Absorção VO & Rápida e completa \\
Biodisponibilidade VO & $90 \%$ \\
Pico plasmático & $1-2 \mathrm{~h}$ \\
Ligação a PP* & $7 \%$ \\
T 1/2 plasmática & $2,6 \mathrm{~h} * *$ \\
Duração de ação & $12 \mathrm{~h}$ \\
Excreção & Renal inalterada $(60 \%)$ \\
\hline PP- proteínas plasmáticas; ** em pacientes com função renal normal. \\
\hline
\end{tabular}

\begin{tabular}{|c|c|}
\hline \multicolumn{2}{|c|}{$\begin{array}{c}\text { Tabela II - Efeitos adversos relatados nos ensaios clínicos } \\
\text { controlados por placebo em pacientes hipertensos tratados com } \\
\text { moxonidina }\end{array}$} \\
\hline \multicolumn{2}{|c|}{ Hipertensos tratados com moxonidina } \\
\hline Efeito adverso & Moxonidina (\%) \\
\hline Xerostomia & $10-15$ \\
\hline Vertigem & $2-7$ \\
\hline Cefaléia & $1-4$ \\
\hline Sonolência & $0-3$ \\
\hline Tosse & 2 \\
\hline Fraqueza/astenia & 1 \\
\hline
\end{tabular}

Efeito na função renal - A administração de moxonidina IV $(0,2 \mathrm{mg})$ em voluntários sadios normotensos, com baixa ingesta de sódio, não teve efeito significativo na TFG, no fluxo sangüíneo renal (FSR) e na excreção urinária de sódio ${ }^{12,23}$. Entretanto, há relatos de um efeito natriurético moderado da moxonidina em pacientes hipertensos, induzido pela ação direta em receptores $\mathrm{I}_{1}$ renais, fato que pode contribuir para o efeito anti-hipertensivo desta droga ${ }^{15}$. Foi descrito, ainda, um aumento da excreção de sódio e água em ratos com nefrectomia unilateral ${ }^{7}$.

Estudos in vivo reforçam esta hipótese pela observação de um efeito natriurético e diurético dose dependente, após a infusão intra-renal de moxonidina, bloqueados por um antagonista $\mathrm{I}_{1}$ (idaxozan), e não por um antagonista $\alpha_{2}^{5,22}$. Em estudo animal comparativo entre nifedipina e moxonidina foi demonstrado que a última, em doses hipotensoras, previne danos renais hipertensivos e causa uma diminuição da espessura das paredes das artérias corticais renais ${ }^{21}$.

Efeito na glicemia - Em estudo controlado por placebo foi demonstrado um efeito anti-hiperglicêmico dose-dependente após tratamento crônico com moxonidina (seis semanas) ${ }^{24}$. Esse efeito pode ser causado indiretamente pela diminuição do tônus simpático, levando à diminuição da lipólise, da glicogenólise, ao aumento do transporte de glicose (diminuição do efeito $\beta_{2}$ ), e à liberação de insulina por estímulo de glicose (diminuição do efeito $\alpha_{2}$ ). Além disso, sugere-se que a moxonidina possa agir de forma direta aumentando a liberação de insulina nas células beta ${ }^{24}$.

Todavia, sabe-se que receptores $I_{1}$ centrais estão envolvidos na regulação dos níveis plasmáticos de glicose e há relatos de que sejam responsáveis pela ação anti-hiperglicêmica da agmatina, outro agonista dos receptores $\mathrm{I}_{1}{ }^{15}$. Desta forma, necessitam-se futuras investigações para esclarecer qual o efeito da moxonidina na glicemia e como ele é mediado.

Outros efeitos - Um estudo em motoristas hipertensos tratados com 0,2 a 0,4mg/dia de moxonidina suprimiu os picos críticos de PA em situações de estresse e não demonstrou alteração no desempenho desses pacientes no trânsito ${ }^{25}$. Observou-se um aumento da atenção e da concentração nesses pacientes ${ }^{25}$. Em ensaios clínicos, a moxonidina não causou alterações significativas nos níveis plasmáticos de eletrólitos, de creatinina, de enzimas hepáticas ou no hematócrito ${ }^{14}$. Verificou-se, ainda, que a administração de moxonidina em animais normotensos, recebendo uma dieta hipercalórica, previne o aparecimento de hipertensão e o aumento da $\mathrm{FC}^{7}$.

\section{Efeitos adversos e interações medicamentosas}

Em estudos controlados por placebo, demonstrou-se uma baixa ocorrência de efeitos adversos, sendo xerostomia o mais observado (tab. II $)^{8,26}$. Essa freqüência de efeitos adversos declinou com o tempo de tratamento, passando de $9,2 \%$ em 12 semanas para $3,8 \%$ em 52 semanas $^{8}$. Não se observou a ocorrência de sedação em estudos placebo- 
controle após dose única ou tratamento crônico com moxonidina ${ }^{26}$. Não há evidências de interações negativas em uso concomitante de moxonidina com digoxina, hidroclorotiazida ou gibenclamida em estudos feitos em humanos ${ }^{16,27}$. Especula-se um possível efeito aditivo com benzodiazepínicos ${ }^{26}$.

\section{Uso clínico}

Dosagem e administração - Em pacientes com hipertensão essencial leve a moderada, recomenda-se uma dose inicial de $0,2 \mathrm{mg} / \mathrm{dia} \mathrm{VO}$, aumentando para $0,4 \mathrm{mg} / \mathrm{dia}$ (ou $0,2 \mathrm{mg} 2 \mathrm{x} / \mathrm{dia}$ ) após três semanas, se o controle da pressão for insuficiente ${ }^{14}$. A dose máxima diária estabelecida para pacientes com função renal normal é de $0,6 \mathrm{mg} / \mathrm{dia} \mathrm{VO}$ e, para pacientes com leve a moderada insuficiência renal, de $0,4 \mathrm{mg} /$ dia VO, tomada junto ou após a refeição ${ }^{14}$.

Indicações e precauções - A moxonidina demonstrou alta eficácia anti-hipertensiva no tratamento de pacientes com HAS essencial leve a moderada, tanto a curto quanto a longo prazos, como previamente descrito ${ }^{8,10,14,17}$. Esta droga não deve ser usada em pacientes com bloqueio cardíaco, arritmias malignas, doença hepática severa ou insuficiência renal grave $(\mathrm{TFG}<30 \mathrm{~mL} / \mathrm{min})^{14}$.

\section{Comparações com outros anti-hipertensivos}

Moxonidina x clonidina - Estudo comparativo placebocontrole demonstrou que ambas possuem eficácia antihipertensiva semelhante e não provocam efeito significativo na FC. Entretanto, a moxonidina apresentou menor freqüência de efeitos adversos e não induziu efeito hipertensivo rebote, nem sedação, observados nos pacientes tratados com clonidina ${ }^{10,17,28}$. Em trabalho realizado em voluntários sadios observou-se que uma dose única de $0,2 \mathrm{mg}$ de moxonidina provocou diminuição da secreção salivar menos intensa do que a clonidina ${ }^{11}$. Há evidências de que essa menor freqüência de efeitos adversos se deva a menor afinidade da moxonidina com receptores a nas glândulas salivares e no locus ceruleus, relacionados, respectivamente, aos efeitos de xerostomia e de sedação ${ }^{5,8,28}$.

Outra diferença significativa entre as duas está na RVP, que é diminuída pela ação da moxonidina e permanece inalterada ou até aumentada (estímulo $\alpha_{1}$ ) por ação da clonidina. Observa-se, também uma diminuição do DC após a administração de clonidina, mas sua preservação com a administração de moxonidina ${ }^{10,29}$. A última foi significativamente melhor tolerada em seis semanas de tratamento com doses equipotentes das drogas ${ }^{14,28}$.

Além disso, ao contrário da clonidina, a ação hipotensiva da moxonidina não se associa com um efeito depressivo na atividade dos nervos respiratórios (frênico e hipoglosso) ${ }^{12}$, ou com efeito rebote após o tratamento ${ }^{30}$. A ação da moxonidina na secreção ácida gástrica ainda não está esclarecida. Há estudos que demonstram uma redução mais potente que a da clonidina ${ }^{31}$, enquanto outros não demonstram alterações significativas nessa secreção durante o uso de moxonidina ${ }^{7}$.
Moxonidina x hidroclorotiazida - Estudos comparativos duplo-cegos controlados por placebo demonstraram efeitos hipotensores equivalentes e incidência de efeitos adversos semelhante entre estas drogas ${ }^{7,10,16,32}$. Há evidência de que a associação terapêutica destes fármacos resulta em uma potencialização de seus efeitos hipotensores, fazendo com que a resposta efetiva ao tratamento aumente de 70 a $88 \%{ }^{7,10,16,32}$.

Moxonidina $\mathbf{x}$ atenolol - Um estudo duplo-cego randomizado sugere que ambos exercem um controle antihipertensivo semelhante após $24 \mathrm{~h}$ da administração $(0,2$ a $0,4 \mathrm{mg} /$ dia de moxonidina e 50 a $100 \mathrm{mg}$ de atenolol). Esse trabalho sugere que moxonidina tem incidência de efeitos adversos igual ou um pouco menor que o atenolol $(21 \% \mathrm{e}$ $26,5 \%$, respectivamente) e uma tolerabilidade no mínimo igual a deste ${ }^{33}$.

Moxonidina x prazosin - Um estudo comparativo demonstrou semelhança na eficácia anti-hipertensiva e na incidência de efeitos adversos destas drogas ${ }^{34}$. Entretanto, o único efeito adverso freqüente relatado com moxonidina foi xerostomia, não sendo verificados diminuição da FC após tratamento agudo ou crônico, hipotensão postural ou sintomas posturais, ao contrário do prazosin ${ }^{34,35}$. Nesse estudo, $10 \%$ dos pacientes do grupo do prazosin abandonaram o tratamento devido aos efeitos adversos de dor no peito, hipotensão postural, vertigem e cefaléia. A moxonidina apresentou ainda uma maior tolerabilidade que o prasozin $(93,3 \% \text { x 53,5\% de tolerância boa/muito boa })^{34}$.

Moxonidina x nifedipina - Um estudo comparativo randomizado, duplo-cego, controlado por placebo demonstrou uma eficácia equivalente e dose dependente entre as drogas no tratamento de hipertensos leve a moderados, com níveis semelhantes de resposta efetiva (70\% para moxonidina e $68 \%$ para nifedipina em doses de 0,2 e $20 \mathrm{mg}$, respectivamente). $\mathrm{O}$ dobro da dose provocou uma resposta $>80 \%$ nos dois grupos ${ }^{20}$. Uma menor frequiência de efeitos adversos foi verificada no grupo da moxonidina $(<30 \% \mathrm{x}$ $40 \%$ ), sendo que xerostomia foi o mais comum. Ao contrário da nifedipina, a moxonidina não causou taquicardia reflexa persistente ${ }^{20}$. Ambas as drogas em doses equipotentes preveniram danos renais associados à hipertensão (glomeruloesclerose, fibrose intersticial e atrofia tubular), embora a nifedipina tenha tendência a induzir hipertrofia glomerular, que pode ser um fator de risco para glomeru3loesclerose ${ }^{36,37}$.

Moxonidina x inibidores da ECA - Estudos comparativos, duplo-cegos entre moxonidina e captopril demonstraram equipotência anti-hipertensiva entre as drogas ${ }^{7,16}$, embora moxonidina tenha se mostrado mais eficaz no controle dos picos hipertensivos matinais ${ }^{16,38}$. Observou-se uma freqüência semelhante de efeitos adversos, caracterizados como leve a moderados ${ }^{7}$. Estudos comparativos entre moxonidina e enalapril apontaram uma redução semelhante na PA em ambas as drogas ${ }^{39}$, embora os níveis de resposta ao tratamento com enalapril (pacientes com PAD <90mmHg) foram levemente superiores $(79 \% \text { x } 59 \%)^{16}$. Nenhuma das drogas apresentou efeito sedativo ou alterações na FC ${ }^{16}$. 


\section{Comentários}

Em inúmeros estudos, foi demonstrada alta eficácia anti-hipertensiva da moxonidina no tratamento de pacientes com HAS essencial leve a moderada, tanto a curto quanto a longo prazos $7,10,14,17,20,33$, sem associação com alterações significativas no DC e na $\mathrm{FC}^{8,10,14}$. Este efeito anti-hipertensivo mostrou-se equipotente ao dos principais grupos de anti-hipertensivos, atualmente empregados no manejo terapêutico da HAS $\mathrm{H}^{7,10,14,17,20,33}$. Além desta eficácia semelhante, a moxonidina não apresentou efeitos adversos posturais (hipotensão postural), ao contrário do prazosin ${ }^{34,35}$, não provocou hipertensão rebote, diferentemente da clonidina e do atenolol ${ }^{33}$, e não desencadeou a taquicardia reflexa observada com o uso de nifedipina ${ }^{20}$. Nesses estudos comparativos, observou-se uma boa tolerabilidade à moxonidina, sendo esta superior à da clonidina ${ }^{17} \mathrm{e}$ à do prazosin ${ }^{34}$, bem como uma frequiência de efeitos adversos no mínimo semelhante à dos demais anti-hipertensivos, sendo inferior à da clonidina (provavelmente pela menor afinidade com os receptores $\left.\mathrm{a}_{2}\right)^{17,28}$, e à da nifedipina ${ }^{20}$. Desses efeitos adversos, o mais freqüentemente descrito foi boca seca ${ }^{8,26}$, não sendo observadas alterações nas funções renal ou hepática ${ }^{14,22,23}$. Demonstraram-se, ainda, uma potencialização do efeito anti-hipertensivo com a associação de moxonidina e hidroclorotiazida ${ }^{7,11,17}$ e uma diminuição do grau de hipertrofia miocárdica após tratamento crônico com moxonidina ${ }^{15}$.

Em suma, verificou-se uma eficácia anti-hipertensiva semelhante entre moxonidina e os demais agentes antihipertensivos, atualmente preconizados, além de uma boa tolerabilidade clínica relacionada a uma baixa freqüência de efeitos adversos. Deste modo, tão logo suas propriedades farmacológicas sejam traduzidas em vantagens clínicas, através de estudos randomizados com desfechos de morbimortalidade, a moxonidina poderárepresentar uma boa alternativa na terapia medicamentosa da HAS.

\section{Referências}

1. Johnston CI-Future management of high blood pressure. J Cardiovasc Pharmacol 1996; 27: S55-60.

2. Kaplan NM - Sistemic hypertension: Mechanism and diagnosis. In: Braunwald E, eds - Heart Disease - A Textbook of Cardiovascular Medicine. $5^{\text {th }}$ ed. Philadelphia: WB Saunders, 1997: 807-39.

3. Kaplan NM-Sistemic hypertension: Therapy. In: Braunwald E, eds - Heart Disease - A Textbook of Cardiovascular Medicine. $5^{\text {th }}$ ed. Philadelphia: WB Saunders, 1997: 840-62.

4. Zwieten PA, Hamilton CA, Julius S, Prichard BNC - Chemistry, Mechanism of Action and Experimental Pharmacology of Moxonidine. : Royal Society Medicine Press 1996; 2: 7-28.

5. Ernsberger P, Damon TH, Graff LM, Schafer SG, Christen MO - Moxonidine, a centrally acting antihypertensive agent, is a selective ligand for $\mathrm{I}_{1}$ - imidazoline sites. J Pharm Exp Ther 1993; 264: 172-82.

6. Reis, DJ - Neurons and receptors in the rostroventrolateral medulla mediating the antihypertensive actions of drugs acting at imidazoline receptors. J Cardiovasc Pharmacol 1996; 27: S11-S18.

7. Dominiak P, Keogh JP, Prichard BNC - Moxonidine. In: Messerli FH, eds - Cardiovascular Pharmacology. $2^{\text {nd }}$ ed. Philadelphia: WB Saunders, 1996: 651-61.

8. Trieb G, Jäger B, Hughes PR, Krosigk GV - Long-term evaluation of the antihypertensive efficacy and tolerability of the orally acting imidazoline $\mathrm{I}_{1}$ receptor agonist moxonidine in patients with mild-to moderate essential hypertension. Eur J Clin Res 1995; 7: 227-40.

9. Ziegler D, Haxhiu MA, Kaan EC, Papp JG, Ernsberger P - Pharmacology of moxonidine, an $\mathrm{I}_{1}$-imidazoline receptor agonist. J Cardiovasc Pharmacol 1996; 27(suppl 3): 26-37.

10. Mitrovic V, Patyna W, Hüting J, Sclepper M - Hemodinamic and neurohumoral effects of moxonidine in patients with essential hypertension. Cardiovasc Drugs Ther 1991; 5: 967-72.

11. Prichard BNC, Graham BR - Effective antihypertensive therapy: blood pressure control with moxonidine. J Cardiovasc Pharmacol 1996; 27: S38-48.

12. Reis DJ - The rostral ventrolateral medulla: a terget of therapy for hypertension. Cardiovascr Risk Factors 1995; 5: 11-8.

13. Ziegler D, Haxhiu MA, Kann EC, Papp IG, Ernsberger P - Pharmacology of moxonidine, na $\mathrm{I}_{1}$ - imidazoline receptor agonist. J Cardiovasc Pharmacol 1996; 27: S26-37.

14. Chrisp P, Faulds D - Moxonidine: A review of its pharmacology, and therapeutic use in essential hypertension. Drugs 1992; 44: 993-1012.

15. Zwieten PA - From a and $b$ to $I_{1}$ : An overview of sympathetic receptors involved in blood pressure control targets for drug treatment. J Cardiovasc Pharmacol 1996; 27: S5-10.

16. Prichard BNC-Clinical Pharmacology of Moxonidine - Royal Society Medicine Press, 1996; 2: 31-45.

17. Schäfers RF, Philipp T - Moxonidine in hipertension treatment - a clinical sum- mary. Cardiovasc Risk Factors 1995; 5: 45-54.

18. Trenk D, Wagner F, Jahnchen E, Planitz V-Pharmacokinetics of moxonidine after single and repeated daily doses in healthy volunteers. J Clin Pharmacol 1987; 27(12): 988-93.

19. Kirch W, Hutt HJ, Planitz V - Pharmacodinamic action and pharmacokinetics of moxonidine after single oral administration in hypertensio patients. J Clin Pharmacol 1990; 30(12): 1088-95.

20. Wolf R - The treatment of hyopertensive patients with a calcium antagonist or moxonidine: A comparison. J Cardiovasc Pharmacol 1992; 20: S42-4.

21. Motz W, Vogt M, Scheler S, Strauer BF-Hypertensive coronary microcirculation - effects of the imidazoline-receptor agonist moxonidine. Cardiovasc Risk Factors $1995 ; 5: 28-32$.

22. Fliser D, Wiecek AJ, Ritz E - Renal hemodynamics. Cardiovasc Risk Factors 1995; 5: 40-4.

23. Mimran A-Antihypertensive therapy and renal function. J Cardiovasc Pharmacol 1996; 27: S19-25.

24. Kaan EC, Bruckner R, Frohly P, Tulp M, Chaefer SG, Ziegler D-Effects of agmatine and moxonidine on glucose metabolism: an integrated approach towards pathophysiological mechanisms in cardiovascular metabolic disorders. Cardiovasc Risk Factors 1995; 5: 19-27.

25. Schmidt U, Frerick JI, Kraft Ket al - Hypertension: A possible risk in road traffic J Cardiovasc Pharmacol 1992; 20: S50-6.

26. Webster J, Koch HF - Aspects of tolerability of centrally acting antihypertensive drugs. J Cardiovasc Pharmacol 1996; 27: S49-54.

27. Morris STW, Reid JL-Moxonidine: a review. J Human Hypertens 1997; 11:629-35.

28. Planitz V -Crossover comparison of moxonidine and clonidine in mild to moderate hypertension. Eur J Clin Pharmacol 1984; 27: 147-52.

29. Rupp H, Maisch B , Brilla CG - Drug withdrawal and rebound hipertension: differential action of the central antihypertensive drugs moxonidine and clonidine. Cardiovascular Drugs Therapy 1996; 10(suppl 1): 251-62.

30. Macphee GA - A comparison of the haemodynamic and behavioral effects of moxonidine and clonidine in normotensive subjects. Br J Clin Pharmacol 1992; 33: 261-7.

31. Glavin GB, Smyth DD - Effects of the selective $\mathrm{I}_{1}$ imidazoline receptor agonist, moxonidine, on gastric secretion and gastric mucosal injury in rats. $\mathrm{Br} \mathrm{J}$ Pharmacol 1995; 114: 751-4.

32. Frei $\mathrm{M}$ - Moxonidine and hydrochlorothiazide in combination: a synergistic antihypertensive effect. J Cardiovasc Pharmacol 1994; 21: 25-8.

33. Prichard BN, Simmons R, Rooks M, Haworth DA, Laws D, Wonnacott S - A Double-blind comparison of moxonidine and atenolol in the management of patients with mild-to-moderate hypertension. J Cardiovasc Pharmacol 1992; 20: S45-9.

34. Plänitz V - Intraindividual comparison of moxonidine and prazosin in hypertensive patients. Eur J Clin Pharmacol 1986; 29: 645-50. 
35. Guthrie GP - Effects os prasozin and clonidine on sympathetic baroreflex function in patients with essential hypertension. J Clin Pharmacol 1983; 23: 348-54.

36. Mall G, Amann K, Ritz E - Modern antihypertensives and nephroprotection. Cardiovasc Risk Factors 1995; 5: 33-9.

37. Irzyniec T, Mall G, Greber D, Ritz E - Beneficial effect of nifedipine and moxonidine on glomerulosclerosis in spontaneously hypertensive rats. A micromorphometric study. Am J Hypertens 1992; 5: 437-43.
38. Kraft K, Vetter H- Twenty-four-hour blood pressure profiles in patient with mildto-moderate hypertension: moxonidine versus captopril. J Cardiovascr Pharmacol 1994; 24(suppl 1): 29-33.

39. Küppers HE, Jäger BA, Luszick JH, Gräve MA, Hughes PR, Kaan EC - Placebocontrolled comparison of the efficacy and tolerability of once-daily moxonidine and enalapril in mild to moderate essential hypertension. J Human Hypertension 1997; 15: 93-7 